\title{
Deficiency in Galectin-3 Promotes Hepatic Injury in CDAA Diet-Induced Nonalcoholic Fatty Liver Disease
}

\author{
Kazuhiro Nomoto, ${ }^{1}$ Takeshi Nishida, ${ }^{1}$ Yuko Nakanishi, ${ }^{1}$ Makoto Fujimoto, ${ }^{2}$ Ichiro Takasaki, ${ }^{3}$ \\ Yoshiaki Tabuchi, ${ }^{3}$ and Koichi Tsuneyama ${ }^{1}$ \\ ${ }^{1}$ Department of Diagnostic Pathology, Graduate School of Medicine and Pharmaceutical Science, University of Toyama, 2630 Sugitani, \\ Toyama 930-0194, Japan \\ ${ }^{2}$ Department of Japanese Oriental Medicine, Graduate School of Medicine and Pharmaceutical Science, University of Toyama, \\ 2630 Sugitani, Toyama 930-0194, Japan \\ ${ }^{3}$ Division of Molecular Genetics Research, Life Science Research Center, University of Toyama, 2630 Sugitani, Toyama 930-0194, Japan
}

Correspondence should be addressed to Kazuhiro Nomoto, knomoto@med.u-toyama.ac.jp

and Takeshi Nishida, dpnishi@med.u-toyama.ac.jp

Received 3 December 2011; Accepted 26 December 2011

Academic Editors: S.-N. Lu and D. Meyre

Copyright (c) 2012 Kazuhiro Nomoto et al. This is an open access article distributed under the Creative Commons Attribution License, which permits unrestricted use, distribution, and reproduction in any medium, provided the original work is properly cited.

\begin{abstract}
Nonalcoholic fatty liver disease (NAFLD) is increasingly recognized as a condition in which excess fat accumulates in hepatocytes. Nonalcoholic steatohepatitis (NASH), a severe form of NAFLD in which inflammation and fibrosis in the liver are noted, may eventually progress to end-stage liver disease. Galectin-3, a $\beta$-galactoside-binding animal lectin, is a multifunctional protein. This protein is involved in inflammatory responses and carcinogenesis. We investigated whether galectin-3 is involved in the development of NASH by comparing galectin-3 knockout $\left(\mathrm{gal}^{-/-}\right)$mice and wild-type $\left(\mathrm{gal}^{+/+}\right)$mice with choline-deficient Lamino-acid-defined (CDAA) diet-induced NAFLD/NASH. Hepatic injury was significantly more severe in the gal $3^{-/-}$male mice, as compared to the gal $3^{+/+}$mice. Data generated by microarray analysis of gene expression suggested that galectin-3 deficiency causes alterations in the expression of various genes associated with carcinogenesis and lipid metabolism. Through canonical pathway analysis, involvement of PDGF and IL-6 signaling pathways was suggested in galectin-3 deficiency. Significant increase of CD14, Fos, and Jun, those that were related to lipopolysaccharide-mediated signaling, was candidate to promote hepatocellular damages in galectin-3 deficiency. In conclusion, galectin-3 deficiency in CDAA diet promotes NAFLD features. It may be caused by alterations in the expression profiles of various hepatic genes including lipopolysaccharide-mediated inflammation.
\end{abstract}

\section{Introduction}

Galectin-3 is a $30-\mathrm{kD}$ mammalian lectin which has a C-terminal carbohydrate-recognition domain and an N-terminal domain comprising multiple repeat sequences rich in glycine, proline, and tyrosine. It is expressed in various cells such as epithelial cells and inflammatory cells. Galectin-3 performs multiple functions, including the regulation of cellto-cell or cell-to-matrix adhesion [1-5], chemoattraction of monocytes/macrophages [6], mediation of pre-mRNA splicing [7], and protection against Fas- and staurosporine-induced apoptosis associated with Bcl-2 expression [8].

During the development of human and mouse embryos, galectin-3 is detectable in most types of cells, including hepatocytes $[9,10]$. In rats, the expression of galectin-3 was found to be rapidly induced in the liver at 9 days after birth and decreased to trace levels in adults [11]. Moreover, very little galectin-3 mRNA was detected in the livers of normal adult rats [12]. In human [13] and mouse livers, galectin-3 expression was not detected in normal hepatocytes, whereas it was found to be prominent in the bile duct epithelial cells and Kupffer cells $[14,15]$. On the other hand, it was found that galectin-3 expression was aberrantly induced in the cytoplasm of the periportal hepatocytes of adult rat liver exhibiting inflammation caused by $\mathrm{CCl}_{4}$ administration [12] and in the hepatocytes surrounding regenerating nodules in human cirrhotic liver [15]. In addition, the results of a previous study showed that the expression of galectin-3 was 
significantly upregulated in activated rat hepatic stellate cells [16]. Galectin-3 expression was also found to be required for the myofibroblast activation and matrix production mediated by transforming growth factor $\beta$ (TGF- $\beta$ ) [17]. Recently, we demonstrated that disrupted galectin-3 expression in male mice leads to the development of nonalcoholic fatty liver disease (NAFLD) and hepatocellular carcinoma (HCC) with liver fibrosis $[18,19]$. Taken together, these results suggest that galectin-3 plays some important roles in liver homeostasis. However, its role in liver pathology, particularly NAFLD, is largely unknown.

NAFLD is increasingly recognized as a liver disorder that may eventually progress to end-stage liver disease, including HCC [20-22]. NAFLD is the preferred term for describing a liver condition that includes a wide spectrum of conditions from simple steatosis to steatohepatitis, advanced fibrosis, and cirrhosis in the liver. The diagnostic criteria for nonalcoholic steatohepatitis (NASH) are continuously evolving and based on histological findings [23, 24]. The most common histopathological features of NASH include hepatocellular steatosis and ballooning degeneration, mixed acute and chronic lobular inflammation, and perisinusoidal fibrosis in the zone 3 regions. Although NASH can be caused by a variety of factors, its occurrence is frequently associated with obesity, type II diabetes, hyperlipidemia, and metabolic syndrome [20,25-27]. The pathogenesis of NASH is poorly understood; however, a "two-hit theory" has been proposed [28]. This theory suggests that, in addition to steatosis (the first "hit"), certain other factor(s) (the second "hit") are required for the development of steatohepatitis. Above all, involvement of lipopolysaccharide is broadly accepted as a candidate of trigger. Recent studies using animal models of NAFLD have also provided new insights into the molecular and physiologic alterations that contribute the first and second hits in the progression of NAFLD to end-stage liver disease $[29,30]$. Various genes and their expression profiles associated with NAFLD have recently been analyzed and identified [31-34].

In the present study, we hypothesized that galectin-3 deficiency increases hepatic injury in mice with NASH induced by a choline-deficient L-amino-acid-defined (CDAA) diet, because galectin-3 is a negative regulator of lipopolysaccharide-mediated inflammation [35]. We report herein that enhancement of lipopolysaccharide signaling pathway involving CD14, Fos, and Jun was observed in galectin3-deficient mice with CDAA diet. Lipopolysaccharide-mediated signaling may not only be a trigger but also be a promoter of NAFLD.

\section{Materials and Methods}

2.1. Animals and Experimental Protocol. Galectin-3 knockout $\left(\mathrm{gal}^{-/-}\right)$mice and their wild-type littermates $\left(\mathrm{gal}^{+/+}\right.$) generated on a CD1 background were generously provided by Dr. Liu's group [36]. The mice were housed under pathogen-free conditions in a temperature-controlled room with a $12 \mathrm{~h} \mathrm{light/dark} \mathrm{illumination} \mathrm{cycle.} \mathrm{All} \mathrm{the} \mathrm{procedures}$ for handling animals were in accordance with the Guide for the Care and Use of Laboratory Animals and approved by the Committee on Animal Experimentation of the University of Toyama. In order to determine the maximum duration required to detect hepatic changes in mice fed the CDAA diet (Dyets, Bethlehem, PA, USA; product no. 518753), a pilot study was conducted in 8- to 10 -week-old male mice fed over a period of $0-12$ weeks to examine the effects of the diet on the serum alanine transaminase (ALT) and aspartate transaminase (AST) levels and on liver steatosis, inflammation, and fibrosis. The severity of liver steatosis, inflammation, and fibrosis observed after 8 weeks of CDAA diet was similar to that observed at 12 weeks; therefore, we determined that maximum duration fed CDAA diet was 8 weeks in this study. Thus, groups of 4 to 6 male gal $3^{-/-}$and $\mathrm{gal}^{+/+}$mice were fed the CDAA diet and sacrificed after 0 , 2,4 , and 8 weeks. The body weights were recorded at the start and end of each experimental period. Blood samples were collected for serum analysis. The livers of the mice were rapidly excised, rinsed in ice-cold saline, and weighed. Liver samples were snap-frozen in liquid nitrogen and maintained at $-80^{\circ} \mathrm{C}$ until analysis. A portion of each liver was fixed in $10 \%$ formalin for histopathological examination.

2.2. Evaluation of Liver Injury. The serum ALT and AST levels were measured using a transaminase CII-Test kit (Wako Pure Chemical Industries, Osaka, Japan) according to the manufacturer's protocol. Liver samples were minced in ice-cold PBS ( $\mathrm{pH} 7.4)$ and homogenized in the buffer at a concentration of $1: 10(w: v)$. After centrifugation at $5,500 \mathrm{rpm}$ for $10 \mathrm{~min}$ at $4^{\circ} \mathrm{C}$, the supernatants were stored for subsequent determination of the total liver triglyceride levels using a commercially available kit (Wako E-test triglyceride kit; Wako Pure Chemical Industries). Data were expressed as milligrams of triglyceride per gram of wet liver weight.

2.3. Histological Analysis. The formalin-fixed liver tissue was processed, and $4-\mu \mathrm{m}$-thick paraffin sections of the liver samples were stained with hematoxylin and eosin for histological analysis. Steatohepatitis was scored by experienced pathologists (K. N. and K. T.) in a blinded manner. Hepatic steatosis was graded as follows according to the percentage of lipid-laden hepatocytes: $0,0 \% ; 1,0-33 \% ; 2,33-67 \%$; 3 , $67-100 \%$. Necroinflammation was graded on the basis of the number and size of the necroinflammatory lesions in representative liver sections as follows: 0 , none; 1 , mild; 2 , moderate; 3 , severe [37].

2.4. RNA Preparation. A liver sample from one mouse in each group fed CDAA diet for 8 weeks was homogenized, and total RNA was extracted using the RNeasy Total RNA Extraction kit (Qiagen, Valencia, CA, USA) and treated with DNase I (RNase-free DNase kit; Qiagen, Valencia, CA, USA) for $15 \mathrm{~min}$ at room temperature to remove residual genomic DNA.

2.5. Affymetrix GeneChip Hybridization. In order to investigate the gene expression profiles, the Affymetrix mouse expression $430 \mathrm{~A}$ array was used. Sample preparation for this 
TABLE 1: Primer pairs and probe sequences used for real-time PCR reactions.

\begin{tabular}{|c|c|}
\hline Target & Sequences \\
\hline$P d g f r b$ & $\begin{array}{l}\text { F: 5' -ACCTGTTCATTTTTGTCACGGATG-3' } \\
\text { R: 5'-TTCTTCTCATGTAGCGTCACCTC-3' } \\
\text { Probe: 5' -6FAM-CGACAATTCCGTGCCGAGTGACAGACC-TAMRA-3' }\end{array}$ \\
\hline Stat 1 & $\begin{array}{l}\text { F: 5' -AGGTGTTGTCAGATCGAACCTTC-3' } \\
\text { R: 5' -CATGCACGGCTGTCGTTCTA-3' } \\
\text { Probe: 5'-6FAM-CTCTTCCAGCAGCTCATTCGGAGCTC-TAMRA-3' }\end{array}$ \\
\hline Csnk2a2 & $\begin{array}{l}\text { F: 5' -AGCCCGGAGGCCCTAGA-3' } \\
\text { R: 5' -TTTGGCGGTCAATCTCTGTTG-3' } \\
\text { Probe: 5'-6FAM-TCTTGACAAGCTCCTGCGGTACGACC-TAMRA-3' }\end{array}$ \\
\hline Fos & $\begin{array}{l}\text { F: 5' -CCTGAGCCCAAGCCATCC-3' } \\
\text { R: 5'-GTCATCAAAGGGTTCTGCCTTC-3' } \\
\text { Probe: 5'-6FAM-CGTTGCTGATGCTCTTGACTGGCTCCA-TAMRA-3' }\end{array}$ \\
\hline Jun & $\begin{array}{l}\text { F: 5' -ACTGCAAAGATGGAAACGACCT-3' } \\
\text { R: 5'-AGCCGTAGGCACCGCTCT-3' } \\
\text { Probe: 5'-6FAM-CGATGCCCTCAACGCCTCGTTC-TAMRA-3' }\end{array}$ \\
\hline$C d 14$ & $\begin{array}{l}\text { F: 5'-CCGAAGCCAGATTGGTCCAG-3' } \\
\text { R: 5'-CACACGCTTTAGAAGGTATTCCAG-3' } \\
\text { Probe: 5'-6FAM-CCGCCGTACAATTCCACATCTGCCG-TAMRA-3' }\end{array}$ \\
\hline GAPDH & $\begin{array}{l}\text { F: 5' -AGGGATGATGTTCTGGGCAG-3' } \\
\text { R: 5'-AGACTGTGGATGGCCCCTC-3' } \\
\text { Probe: 5' -6FAM-ACGGCCATCACGCCACAGCTTT-TAMRA-3' }\end{array}$ \\
\hline
\end{tabular}

Pdgfrb: platelet-derived growth factor receptor, beta polypeptide; Stat1: signal transducer and activator of transcription 1; Csnk2a2: casein kinase 2, alpha prime polypeptide; Fos: FBJ osteosarcoma oncogene; GAPDH: glyceraldehyde-3-phosphate dehydrogenase.

analysis was performed according to the procedure described in the Affymetrix GeneChip Expression technical manual. Briefly, $5 \mu \mathrm{g}$ of total RNA was used to synthesize double-stranded cDNA by using a GeneChip Expression 3'Amplification Reagents One-cycle cDNA Synthesis Kit (Affymetrix, Santa Clara, CA, USA). Biotin-labeled cRNA was then synthesized using GeneChip Expression 3'-Amplification Reagents for IVT Labeling (Affymetrix). After fragmentation, the biotinylated cRNA was hybridized to a GeneChip array at $45^{\circ} \mathrm{C}$ for $16 \mathrm{~h}$. The chip was washed, stained with streptavidin-phycoerythrin, and scanned with a GeneChip scanner 3000 (Affymetrix), and the results were analyzed using the GeneChip Analysis Suite Software (Affymetrix). Hybridization intensity data were represented as presence/absence calls for each gene, and the differences in gene expression between experiments were detected by comparison analysis. The data were further analyzed using GeneSpring version 7.3 (Silicon Genetics, Redwood City, CA, USA) to extract significantly expressed genes and determine their ontology, including biological processes, cellular components, and molecular functions. Genes were considered upregulated or downregulated if there was 2-fold or greater difference in their expression between the gal $3^{-/-}$and the $\mathrm{gal} 3^{+/+}$mice.

2.6. Functional Network, Gene Ontology, and Canonical Pathway Analyses. The genes identified by GeneSpring were used for functional network and gene ontology analyses. Gene accession numbers were imported into the Ingenuity Pathway Analysis version 3.1 (IPA) software (Ingenuity Systems, Mountain View, CA, USA), and the gene products were categorized using the software on the basis of their hepatic location and cellular localization and the reported or suggested biochemical, biological, and molecular functions. The genes were then mapped to the genetic networks available in the Ingenuity database on the basis of their scorewise ranking, and the probability that a collection of genes is to the same as or greater than the number of genes in a network was a matter of chance. A score of 3 obtained randomly indicates that there is a $1 / 1000$ chance that the identified genes are a part of the network. Therefore, scores of 3 or higher have a $99.9 \%$ confidence level of not having been generated randomly. This score was used as the cutoff for identifying gene networks.

2.7. Real-Time PCR. Six genes included in the highly significant canonical pathways (PDGF signaling and IL-6 signaling) were chosen for further validation by real-time PCR. Total RNA was isolated from the liver samples using Isogen reagent (Nippon Gene, Toyama, Japan). cDNA was synthesized from $400 \mathrm{ng}$ of hepatic mRNA by using TaqMan Reverse Transcription Reagents (Applied Biosystems, Tokyo, Japan). PCR reactions and analyses were carried out using an Mx3000P QPCR System (Stratagene, La Jolla, CA, USA) with denaturation for $10 \mathrm{~min}$ at $95^{\circ} \mathrm{C}$, followed by 50 PCR cycles of denaturation at $95^{\circ} \mathrm{C}$ for $30 \mathrm{~s}$, annealing at $55^{\circ} \mathrm{C}$ for $1 \mathrm{~min}$ and extension at $72^{\circ} \mathrm{C}$ for $30 \mathrm{~s}$. All primers and probes used for analysis were designed at Nipppon EGT (Toyama, Japan). The primer and probe sequence details are given in Table 1. The amount of mRNA was calculated using glyceraldehydes3-phosphate dehydrogenase $(G A P D H)$ as the endogenous control. 
TABLE 2: Effect of CDAA diet on serum ALT, serum AST, and liver triglyceride levels in the gal3 $3^{+/+}$and gal3 ${ }^{-/-}$mice.

\begin{tabular}{|c|c|c|c|c|c|}
\hline & & \multicolumn{4}{|c|}{ CDAA diet consumption } \\
\hline & & $0 \mathrm{wk}$ & $2 \mathrm{wk}$ & $4 \mathrm{wk}$ & $8 \mathrm{wk}$ \\
\hline \multirow{2}{*}{ Serum ALT (IU/L) } & Gal- $3^{+/+}$ & $26.9 \pm 4.3$ & $48.2 \pm 9.8$ & $89.9 \pm 33.3$ & $64.2 \pm 17.5$ \\
\hline & Gal-3-/- & $19.7 \pm 1.0$ & $109.6 \pm 18.3^{*}$ & $151.2 \pm 45.6$ & $260.9 \pm 101.6$ \\
\hline \multirow{2}{*}{ Serum AST (IU/L) } & $\mathrm{Gal}-3^{+/+}$ & $78.6 \pm 7.6$ & $80.7 \pm 10.7$ & $117.6 \pm 27.3$ & $75.7 \pm 9.5$ \\
\hline & Gal-3 ${ }^{-/-}$ & $78.5 \pm 11.5$ & $114.9 \pm 15.1$ & $153.7 \pm 26.6$ & $192.1 \pm 39.9^{*}$ \\
\hline \multirow{2}{*}{ Liver triglyceride $(\mathrm{mg} / \mathrm{g})$} & Gal- $3^{+/+}$ & $13.3 \pm 0.5$ & $51.7 \pm 3.4$ & $64.9 \pm 5.6$ & $75.1 \pm 1.7$ \\
\hline & Gal-3-/- & $15.7 \pm 1.0$ & $58.1 \pm 4.1$ & $66.0 \pm 4.0$ & $88.1 \pm 10.7$ \\
\hline
\end{tabular}

Results are expressed as mean \pm SEM of the values from $4-6$ mice in each group. ${ }^{*} P<0.05$ in comparison with gal3 ${ }^{+/+}$mice. CDAA: choline-deficient Lamino-acid-defined diet; ALT: alanine aminotransferase; AST: aspartate aminotransferase.

2.8. Statistical Analysis. The values were expressed as means \pm SEM. The means were compared using the Mann-Whitney $U$-test. $P<0.05$ was considered statistically significant.

\section{Results}

3.1. Effect of CDAA Diet on Liver Injury in $\mathrm{Gal3}^{-/-}$Mice. Mice fed the CDAA diet showed an increase in serum ALT and AST levels and liver triglyceride levels at week 2, and the levels remained elevated up to week 8. The serum ALT and AST levels were determined to be significantly higher in the gal $3^{-/-}$mice, as compared to the gal $3^{+/+}$mice after either 2 or 8 weeks of subjection to the CDAA diet $(P<0.05)$ (Table 2), whereas the increase in the liver triglyceride levels in the gal3 $3^{-/-}$mice was not significant difference compared to the $\mathrm{gal} 3^{+/+}$mice.

\subsection{Effect of CDAA Diet on Hepatic Morphology in $\mathrm{Gal3}^{-1-}$} Mice. After 2 weeks of CDAA diet consumption, macrovesicular steatosis involving lobular zones 1 and 2 was observed for both mice. The hepatocytes were swollen with mildto-moderate micro- and macrovesicular steatosis and mild lobular and portal necroinflammation, which were more severe in the gal3 ${ }^{-/-}$mice (Figures $1(\mathrm{c})$ and $1(\mathrm{~d})$ ). In 4 weeks, steatosis was further severe in the gal $3^{-/-}$mice (Figures $1(\mathrm{e})$ and $1(\mathrm{f}))$. The degree of steatosis after 2 or 4 weeks was significantly higher in the gal $3^{-/-}$mice $(P<0.05)$ (Table 3$)$, and the degree of necroinflammation after 2 weeks was also significantly higher in the gal $3^{-/-}$mice $(P<0.05)$ (Table 3$)$. In 8 weeks, both mice showed further severe steatosis and moderate lobular and portal necroinflammation (Figures $1(\mathrm{~g})$ and $1(\mathrm{~h}))$.

\subsection{Affymetrix GeneChip Analysis of Genes in CDAA Diet-} Induced NAFLD. The expression of 22,625 mouse transcripts was detected using the Affymetrix mouse expression $430 \mathrm{~A}$ array. In analysis of liver samples from the mice after 8 weeks of the CDAA diet, we identified 460 probes whose expression was upregulated by $>2$-fold and 185 probes whose expression was downregulated by $>2$-fold in gal $^{-/-}$mice compared to the gal $3^{+/+}$mice.

3.4. Functional Network and Gene Ontology Analysis. To further refine our study, we investigated the biological inter- actions of the 645 genes identified by the GeneChip analysis using the IPA tool. We found that these 645 genes mapped to genetic networks and had functional relationships. We also identified 37 genetic networks in the livers of the gal $3^{-/-}$ mice. Of these 37, 24 were highly significant in that they included more of the identified genes than expected by chance. Networks with high scores $(>15)$ included more than half the identified genes (these are listed in Table 4), which are associated with cancer, cell death, cellular assembly and organization, cellular function and maintenance, organismal injury and abnormalities, carbohydrate and lipid metabolism, and so forth. We also performed gene ontology analysis using the IPA tool and identified 73 categories as significant (data not shown).

3.5. Canonical Pathway Analysis. In the canonical pathway analysis, we identified 2 significant pathways (PDGF signaling and IL- 6 signaling pathways) in the liver samples of gal3 ${ }^{-1-}$ mice at after 8 weeks on the CDAA diet (Figure 2).

3.6. Validation of Gene Expression with Real-Time PCR. In order to confirm the results of the Ingenuity pathway analysis, we measured the expression of 6 genes included in the highly significant canonical pathways. The genes in the PDGF signaling and IL-6 signaling pathway include Pdgfrb, Stat1, Csnk2a2, Fos, Jun, and Cd14. mRNAs for Fos, Jun, and $C d 14$ were significantly increased in gal3 ${ }^{-/-}$mice fed the CDAA diet compared to the gal $3^{+/+}$mice $(P<$ 0.05) (Figure 3). Interestingly, these were key molecules of lipopolysaccharide-mediated inflammation. mRNAs for $P d g f r b$, Stat1, and Csnk2a2 were also increased, but not significantly.

\section{Discussion}

Our results demonstrated that a deficiency in galectin-3 resulted in an increase in hepatic injury in CDAA diet-induced NAFLD. Additionally, the data we obtained by microarray analysis of gene expression suggested that galectin3 deficiency, in the background of CDAA diet-induced NAFLD/NASH, may cause alterations in the expression of various genes associated with cancer, cell death, cellular assembly and organization, cellular function and maintenance, organismal injury and abnormalities, and carbohydrate and 


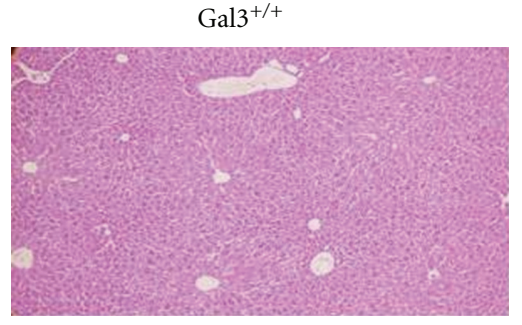

(a)

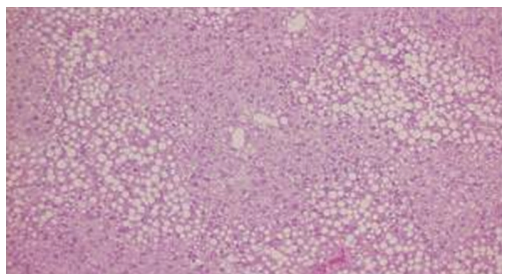

(d)

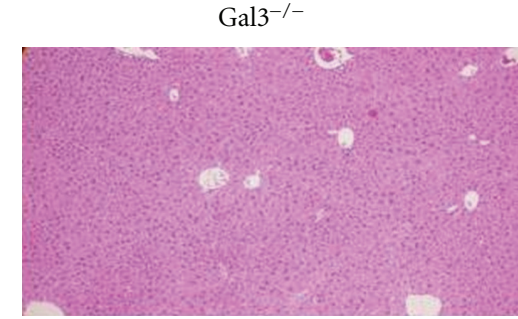

(b)

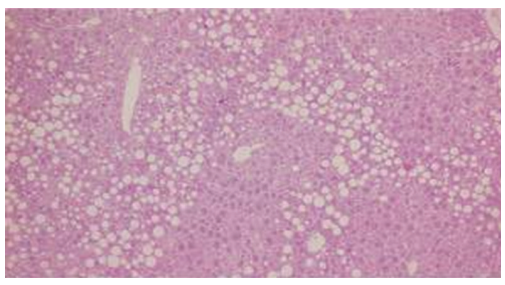

(e)

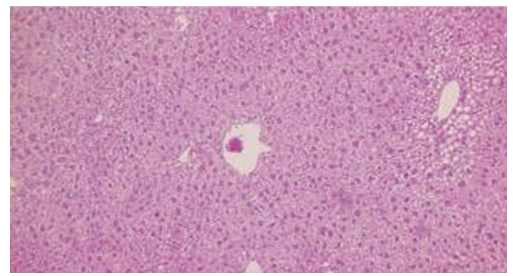

(c)

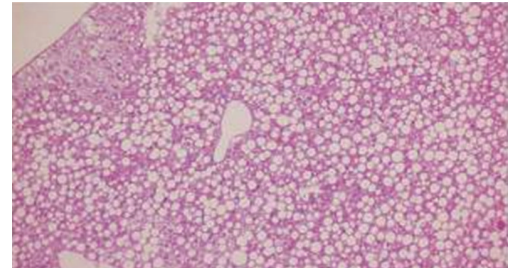

(f)

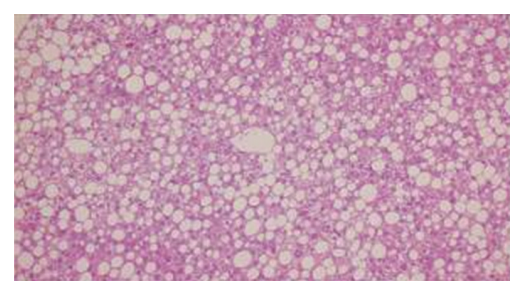

(g)

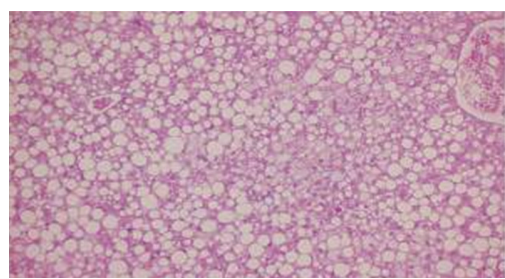

(h)

FIGURE 1: Representative photomicrographs showing the effect of choline-deficient L-amino-acid-defined (CDAA) diet on liver histology in $\mathrm{gal}^{+/+}$and gal3 ${ }^{-/-}$mice. (a) and (b) are week 0 of CDAA diet (Control). In both mice, histopathological differences were not observed. (c) and (d) are week 2. (e) and (f) are week 4. (g) and (h) are week 8 (hematoxylin and eosin staining, original magnification $\times 100$ ).

TABLE 3: Effect of CDAA diet and galectin-3 deficiency on the severity of hepatic steatosis and necroinflammation.

\begin{tabular}{|c|c|c|c|c|c|}
\hline & & \multicolumn{4}{|c|}{ CDAA diet consumption } \\
\hline & & $0 \mathrm{wk}$ & $2 \mathrm{wk}$ & $4 \mathrm{wk}$ & $8 \mathrm{wk}$ \\
\hline \multirow{2}{*}{ Steatosis } & $\mathrm{Gal}^{+/+}$ & $0.0 \pm 0.0$ & $0.6 \pm 0.5$ & $1.5 \pm 0.6$ & $2.3 \pm 1.0$ \\
\hline & $\mathrm{Gal}^{-/-}$ & $0.0 \pm 0.0$ & $2.0 \pm 0.7^{*}$ & $2.8 \pm 0.5^{*}$ & $2.8 \pm 0.5$ \\
\hline \multirow{2}{*}{ Necroinflammation } & $\mathrm{Gal}^{+/+}$ & $0.0 \pm 0.0$ & $0.6 \pm 0.5$ & $1.5 \pm 0.6$ & $2.0 \pm 0.0$ \\
\hline & $\mathrm{Gal3}^{-/-}$ & $0.0 \pm 0.0$ & $1.8 \pm 0.4^{*}$ & $1.8 \pm 0.5$ & $2.0 \pm 0.0$ \\
\hline
\end{tabular}

The severity of both hepatic steatosis and necroinflammation was scored on a scale of 0-3, as described in Materials and Methods. Results are expressed as mean \pm SEM of the values from $4-6$ mice in each group. ${ }^{*} P<0.05$ in comparison with gal $3^{+/+}$mice.

lipid metabolism. On the basis of our results, we were able to propose several potential mechanisms underlying the increase in hepatic injury observed in gal $3^{-1-}$ mice. The first possible mechanism is an increase in hepatocyte apoptosis. Previous studies have demonstrated that hepatocyte apoptosis was significantly increased in NASH [38-40]. Furthermore, Yang et al. reported on the protective effects of galectin-3 against Fas- and staurosporine-induced apoptosis associated with $\mathrm{Bcl}-2$ expression [8]. In our study, microarray analysis revealed that the expression of genes associated with cell death significantly increased in the gal $3^{-/-}$mice compared to the gal3 $3^{+/+}$mice. These data suggest that galectin-3 plays an important role as an antiapoptotic protein in hepatocytes affected by CDAA diet-induced NAFLD.

In the canonical pathway analysis and validation of gene expression with real-time PCR, we observed that the expression of components of the PDGF and IL-6 signaling pathways were significantly amplified in the liver of the gal3 ${ }^{-1-}$ mice after 8 weeks of the CDAA diet. As members of the PDGF ligand family are known to play important roles in cellular proliferation and migration, this finding provided second possible mechanism behind the increased hepatic injury observed in gal $3^{-/-}$mice. We recently reported a case of severe fibrosis affecting multiple organs caused by neonatal PDGF overproduction [41]. In this case, the PDGF- $\beta$ receptor was found to be overexpressed in all the fibrotic organs, including the liver. Moreover, a previous study showed that PDGF-C transgenic mice had enlarged livers associated with increased fibrosis, steatosis, cell dysplasia, and HCC [42]. These studies indicated that the overexpression of PDGF and its receptor induces a number of profibrotic pathways, suggesting that this growth factor acts 
TABLE 4: Genetic networks with high scores $(>15)$ in gal3 ${ }^{-/-}$mice with CDAA diet-induced NAFLD.

\begin{tabular}{|c|c|c|c|c|}
\hline Network & Genetic in Ingenuity networks ${ }^{\mathrm{a}}$ & Score & Focus genes & Top functions \\
\hline 1 & $\begin{array}{l}\text { ACTG1, ANXA2, AP3D1, CA3, CD36, CD151, } \\
\text { CEACAM1, COTL1, CTSB, DNMT3A, ETS2, FOS, } \\
\text { GAS1, GSTA5, GSTM3, HNRPAB, IFRD1, ITGA2B, } \\
\text { JUN, MT1A, MT2A, MTF1, NMI, PBX2, RBM39, } \\
\text { RBP1, RPL39, S100A10, SERPINA3G, SLPI, SPP1, } \\
\text { TGM2, TMED10, TTRAP, TUBB2A }\end{array}$ & 49 & 35 & $\begin{array}{l}\text { Cancer, cell death, cellular assembly and } \\
\text { organization }\end{array}$ \\
\hline 2 & $\begin{array}{l}\text { BAG3, BMPR1A, C5ORF13, CD2AP, COL1A1, } \\
\text { COL3A1, DIO1, DNAJB1, HDAC3, HSP90AA1, } \\
\text { HSPA8, HSPA1A, HSPH1, IFIH1, IRF3, KLF6, KPNA4, } \\
\text { LIFR, MBD1, P4HA1, PDGFC, PDGFRB, PPP1CB, } \\
\text { PTPN2, RHOB, RND3, ROCK1, SERPINH1, STAT1, } \\
\text { TANK, TBK1, TFRC, TGFBR2, TGTP, USP18 }\end{array}$ & 49 & 35 & $\begin{array}{l}\text { Cellular function and maintenance, } \\
\text { dermatological diseases and conditions, } \\
\text { organismal injury and abnormalities }\end{array}$ \\
\hline 3 & $\begin{array}{l}\text { ABCB11, ABCC3, BRD8, CDC20, CDH1, CPT1A, } \\
\text { CSNK2A2, CYP3A5, CYP4A22, CYP7A1, DLG1, } \\
\text { DNAJB4, DUSP6, FOXA1, G6PC, GADD45A, } \\
\text { GADD45G, GCK, HNRPH2, IGHM, INSIG2, IQGAP1, } \\
\text { LCN2, LGALS1, MAP3K4, PCK1, PPARGC1B, PTPRC, } \\
\text { RXRA, SCD, SMAD4, SQLE, SSRP1, SUB1, TMSB4X }\end{array}$ & 49 & 35 & Carbohydrate metabolism, cell death, cancer \\
\hline 4 & $\begin{array}{l}\text { ACSL4, AFP, ALDOC, ARF6, ARMET, CES1, COL5A2, } \\
\text { DUSP6, EGLN3, ENPP1, ERBB2, GJA1, HAS2, HGF, } \\
\text { HIF1A, HRAS, LCN2, LDHA, LGALS1, NDST1, NID1, } \\
\text { PDLIM1, PGAM1, PGF, PLOD1, PQLC1, PRDX2, } \\
\text { RHOB, S100A4, SPRY2, SQLE, TMSB4X, TSC22D1, } \\
\text { TSC22D4, UCRC }\end{array}$ & 20 & 21 & $\begin{array}{l}\text { Cancer, cellular movement, reproductive } \\
\text { system disease }\end{array}$ \\
\hline 5 & $\begin{array}{l}\text { ABCB1, ATF3, ATR, CASP4, CCNG1, CEBPD, CHUK, } \\
\text { CSF1R, DUSP1, FOXO3A, GBP4, GPIAP1, GSTM5, } \\
\text { H2AFZ, HSP90AA1, IFIT1L, ISG15, MCM2, MCM3, } \\
\text { MCM4, MCM6, MDM2, NOX4, PDCD6IP, POLB, } \\
\text { PPP2R4, RALGPS2, SON, SPP1, STRN3, SUMO1, } \\
\text { TANK, TP53, UBQLN1, WT1 }\end{array}$ & 19 & 20 & Cancer, cell cycle, reproductive system disease \\
\hline 6 & $\begin{array}{l}\text { ANXA2, CD14, CIITA, COL4A2, COL6A1, COL6A2, } \\
\text { COL6A3, CREB1, ELN, EMILIN1, FGF10, GNAS, } \\
\text { IRF7, LDHA, MAPKAPK2, MFI2, NCL, OAT, OPRM1, } \\
\text { PCSK1, PITPNB, PLEKHC1, PTHLH, S100A11, SCD, } \\
\text { SCOTIN, SEC63, SFTPB, SSTR2, TGFB1, TGM1, TH, } \\
\text { TMEM123, TNFRSF10A, VNN1 }\end{array}$ & 17 & 19 & $\begin{array}{l}\text { Genetic disorder, skeletal and muscular } \\
\text { disorders, dermatological diseases and } \\
\text { conditions }\end{array}$ \\
\hline 7 & $\begin{array}{l}\text { ACVR1, AKAP12, CD14, CD53, CEACAM5, CEBPA, } \\
\text { CPB2, CSF1R, CYP3A5, DDIT3, EPAS1, FCGR1A, } \\
\text { GAL, HP, IGSF1, IK, IL6, INHBA, INHBC, INHBE, } \\
\text { ITGA2B, LGALS4, LOX, PGD, PSCDBP, PTPRC, } \\
\text { PTPRZ1, SAA1, SLCO1A1, SOAT1, SPBC25, STAR, } \\
\text { TRIB1, UCP1, USP52 }\end{array}$ & 17 & 19 & $\begin{array}{l}\text { Lipid metabolism, molecular transport, small } \\
\text { molecule biochemistry }\end{array}$ \\
\hline 8 & $\begin{array}{l}\text { ALDOB, APOM, ARPC4, ATP6V1E1, CD14, CD36, } \\
\text { CEBPB, CPS1, CSF3, CSTB, CXCL6, CYP24A1, } \\
\text { CYP4A22, CYP7A1, ELOVL3, FABP3, FGF19, } \\
\text { HCA112, LCN2, LEP, MBOAT5, NFKBIZ, NRG1, } \\
\text { ORM1, PDK4, PPARA, PTGER4, SCD, SLC10A2, } \\
\text { SLC20A1, SRXN1, TNFSF11, TRAF6, UGP2, ZNF274 }\end{array}$ & 17 & 19 & $\begin{array}{l}\text { Carbohydrate metabolism, molecular } \\
\text { transport, small molecule biochemistry }\end{array}$ \\
\hline
\end{tabular}

${ }^{\text {a }}$ Genes colored red or green are those identified by microarray analysis as upregulated and downregulated, respectively.

as an initiator of fibrosis [42]. We speculated that galectin3 deficiency might lead to the higher expression of genes related to the PDGF signaling pathway and consequently accelerate the development of various pathological conditions such as fibrosis and carcinogenesis in patients with NAFLD/NASH.

In a recent landmark study, Cai et al. demonstrated that hepatic IL-6, a major proinflammatory cytokine, is markedly expressed in animal models of NAFLD [43]. Moreover, Wieckowska and coworkers found significant increase in hepatic IL-6 expression in NASH patients [44]. In the present study, the expression of genes involved in the IL- 6 signaling pathway was significantly higher in the gal $3^{-/-}$mice. These data suggest that galectin-3 deficiency may accelerate the hepatic IL-6 signaling pathway, leading to an increased inflammatory response, steatosis, and, finally, steatohepatitis 


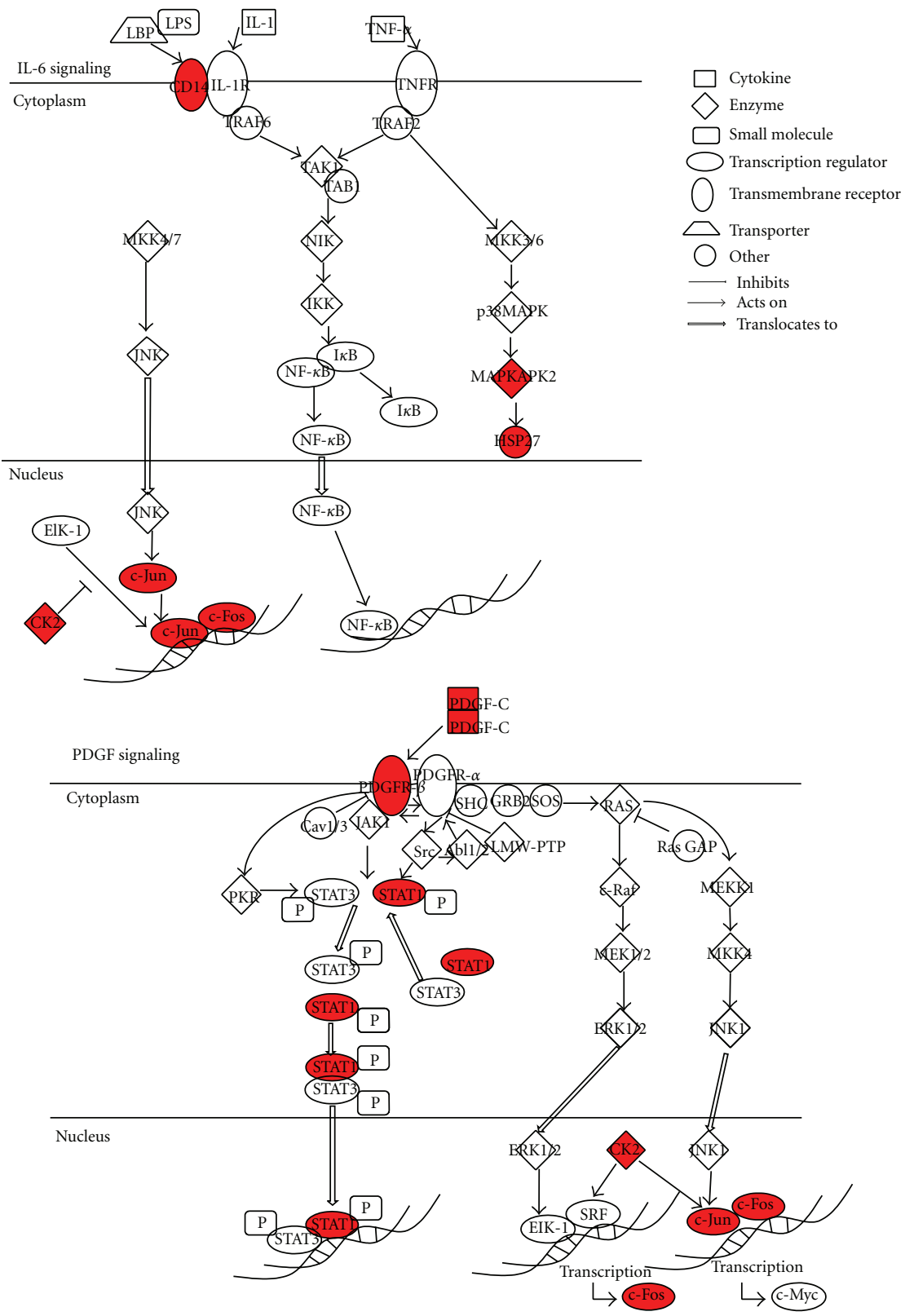

FIGURE 2: The canonical pathways of the PDGF and IL-6 signaling. These pathways were significantly identified in the complete data set. The genes indicated in red are those that were upregulated; those indicated in white are not user specified but were incorporated into the network since they shared functional relationships with the upregulated genes.

thus, providing a third possible mechanism behind the increased hepatic injury observed in gal $3^{-1-}$ mice. Amongst related molecules of IL-6 signaling pathway, CD14, Fos, and Jun were significantly increased in gal $3^{-/-}$mice. These molecules were key molecules of lipopolysaccharide signaling. It is broadly accepted that lipopolysaccharide is one of an important trigger of NAFLD. In 8 weeks of CDAA diet, there are no significant pathological changes between gal3 $3^{-/-}$and gal $^{+/+}$mice, while the titer of ALT and AST was quite high in gal3 $^{-/-}$mice. We hypothesized that lipopolysaccharidemediated signaling may not only be a trigger but also be a promoter of NAFLD. Galectin-3, which is a negative regulator of lipopolysaccharide-mediated inflammation, may be effective to inhibit disease promotion of NAFLD/NASH. Treatment of NAFLD by recruiting galectin-3 should be examined using animal model of NAFLD in further study.

Previous studies have demonstrated that NASH can progress to cirrhosis and eventually to $\operatorname{HCC}[45,46]$; however, its carcinogenetic mechanisms are largely unknown. Although several animal models have been proposed in order to understand the pathogenesis of NAFLD [47], the possible neoplastic transformation that characterizes endstage NASH has been developed in only a few models, including aged gal3 $3^{-/-}$mice $[18,48]$. In the functional network and gene ontology analysis, we identified networks with high scores $(>15)$, wherein more than half of the identified 


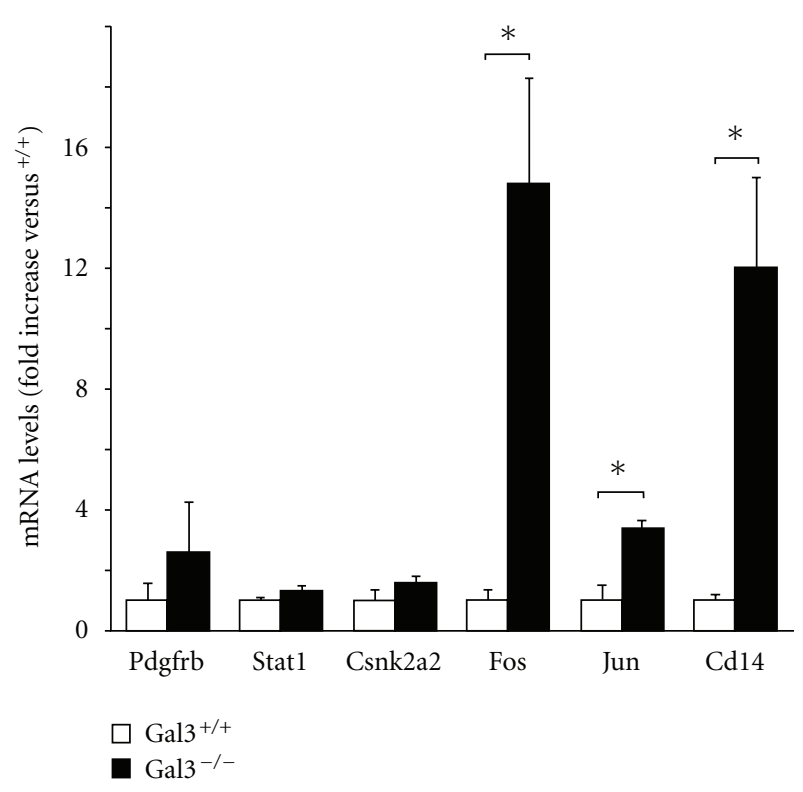

Figure 3: Comparison with mRNA levels of the altered genes in the significant canonical pathway (PDGF signaling and IL-6 signaling). These levels were determined by real-time PCR, normalized to glyceraldehydes-3-phosphate dehydrogenase (GAPDH), and expressed as fold induction relative to the gal $3^{+/+}$mice. The values are expressed as mean \pm SEM of four mice in each group. $\left({ }^{*} P<0.05\right)$.

genes were associated with cancer, cell death, cellular assembly and organization, cellular function and maintenance, organismal injury and abnormalities, and carbohydrate and lipid metabolism. Therefore, we hypothesize that galectin-3 deficiency under the condition of NAFLD/NASH contributes to liver carcinogenesis.

In conclusion, our study demonstrated that a deficiency in galectin-3 increases hepatic injury in mice with CDAA diet-induced NAFLD/NASH. Recruitment of galectin-3 may be a new target of the treatment of NAFLD/NASH.

\section{Authors' Contribution}

K. Nomoto and T. Nishida contributed equally to this work; K. Nomoto, Y. Nakanishi, M. Fujimoto and K. Tsuneyama designed the research and performed the majority of experiments; I. Takasaki and Y. Tabuchi contributed genechip analytic tool and analyzed the data; K. Nomoto, T. Nishida, and K. Tsuneyama wrote the paper.

\section{Acknowledgments}

The authors especially thank Professor Masakiyo Sasahara for his expert opinion regarding PDGF signaling. They also thank Tokimasa Kumada, Hideki Hatta, and Takako Matsushima for their excellent technical assistance and Yukari Inoue for her support while preparing this paper. This study was supported by Grants-in-Aid for Scientific Research (16790223 and 18590324) from the Japanese Ministry of Education, Science, Sports and Culture, Uehara Memorial Foundation, and the 21 st century COE program in Japan.

\section{References}

[1] I. Kuwabara and F. T. Liu, "Galectin-3 promotes adhesion of human neutrophils to laminin," Journal of Immunology, vol. 156, no. 10, pp. 3939-3944, 1996.

[2] H. Inohara, S. Akahani, K. Koths, and A. Raz, "Interactions between galectin-3 and Mac-2-binding protein mediate cellcell adhesion," Cancer Research, vol. 56, no. 19, pp. 4530-4534, 1996.

[3] J. Ochieng, P. Warfield, B. Green-Jarvis, and I. Fentie, "Galectin-3 regulates the adhesive interaction between breast carcinoma cells and elastin," Journal of Cellular Biochemistry, vol. 75, no. 3, pp. 505-514, 1999.

[4] S. Sato, N. Ouellet, I. Pelletier, M. Simard, A. Rancourt, and M. G. Bergeron, "Role of galectin-3 as an adhesion molecule for neutrophil extravasation during streptococcal pneumonia," Journal of Immunology, vol. 168, no. 4, pp. 1813-1822, 2002.

[5] J. Zou, V. V. Glinsky, L. A. Landon, L. Matthews, and S. L. Deutscher, "Peptides specific to the galectin-3 carbohydrate recognition domain inhibit metastasis-associated cancer cell adhesion," Carcinogenesis, vol. 26, no. 2, pp. 309-318, 2005.

[6] H. Sano, D. K. Hsu, L. Yu et al., "Human galectin-3 is a novel chemoattractant for monocytes and macrophages," Journal of Immunology, vol. 165, no. 4, pp. 2156-2164, 2000.

[7] S. F. Dagher, J. L. Wang, and R. J. Patterson, "Identification of galectin-3 as a factor in pre-mRNA splicing," Proceedings of the National Academy of Sciences of the United States of America, vol. 92, no. 4, pp. 1213-1217, 1995.

[8] R. Y. Yang, D. K. Hsu, and F. T. Liu, "Expression of galectin3 modulates T-cell growth and apoptosis," Proceedings of the National Academy of Sciences of the United States of America, vol. 93, no. 13, pp. 6737-6742, 1996.

[9] D. Fowlis, C. Colnot, M. A. Ripoche, and F. Poirier, "Galectin3 is expressed in the notochord, developing bones, and skin of the postimplantation mouse embryo," Developmental Dynamics, vol. 203, no. 2, pp. 241-251, 1995.

[10] F. A. Van Den Brûle, P. L. Fernandez, C. Buicu et al., "Differential expression of galectin-1 and galectin-3 during first trimester human embryogenesis," Developmental Dynamics, vol. 209, no. 4, pp. 399-405, 1997.

[11] R. F. Cerra, M. A. Gitt, and S. H. Barondes, "Three soluble rat $\beta$-galactoside-binding lectins," Journal of Biological Chemistry, vol. 260, no. 19, pp. 10474-10477, 1985.

[12] K. Yamazaki, A. Kawai, M. Kawaguchi et al., "Simultaneous induction of galectin-3 phosphorylated on tyrosine residue, p21WAF1/Cip1/Sdil, and the proliferating cell nuclear antigen at a distinctive period of repair of hepatocytes injured by CCl4," Biochemical and Biophysical Research Communications, vol. 280, no. 4, pp. 1077-1084, 2001.

[13] T. Ota, T. Takamura, S. Kurita et al., "Insulin resistance accelerates a dietary rat model of nonalcoholic steatohepatitis," Gastroenterology, vol. 132, no. 1, pp. 282-293, 2007.

[14] T. J. Flotte, T. A. Springer, and G. J. Rhorbecke, "Dendritic cell and macrophage staining by monoclonal antibodies in tissue sections and epidermal sheets," American Journal of Pathology, vol. 111, no. 1, pp. 112-124, 1983.

[15] D. K. Hsu, C. A. Dowling, K. C. G. Jeng, J. T. Chen, R. Y. Yang, and F. T. Liu, "Galectin-3 expression is induced in cirrhotic liver and hepatocellular carcinoma," International Journal of Cancer, vol. 81, no. 4, pp. 519-526, 1999.

[16] N. Maeda, N. Kawada, S. Seki et al., "Stimulation of proliferation of rat hepatic stellate cells by galectin-1 and galectin-3 through different intracellular signaling pathways," Journal of Biological Chemistry, vol. 278, no. 21, pp. 18938-18944, 2003. 
[17] N. C. Henderson, A. C. Mackinnon, S. L. Farnworth et al., "Galectin-3 regulates myofibroblast activation and hepatic fibrosis," Proceedings of the National Academy of Sciences of the United States of America, vol. 103, no. 13, pp. 5060-5065, 2006.

[18] Y. Nakanishi, K. Tsuneyama, K. Nomoto et al., "Nonalcoholic steatohepatitis and hepatocellular carcinoma in galectin-3 knockout mice," Hepatology Research, vol. 38, no. 12, pp. 1241-1251, 2008.

[19] K. Nomoto, K. Tsuneyama, H. O. Abdel Aziz et al., "Disrupted galectin-3 causes non-alcoholic fatty liver disease in male mice," Journal of Pathology, vol. 210, no. 4, pp. 469-477, 2006.

[20] A. E. Reid, "Nonalcoholic steatohepatitis," Gastroenterology, vol. 121, no. 3, pp. 710-723, 2001.

[21] P. Angulo, "Medical progress: nonalcoholic fatty liver disease," New England Journal of Medicine, vol. 346, no. 16, pp. 12211231, 2002.

[22] B. A. Neuschwander-Tetri and S. H. Caldwell, "Nonalcoholic steatohepatitis: summary of an AASLD Single Topic Conference," Hepatology, vol. 37, no. 5, pp. 1202-1219, 2003.

[23] E. M. Brunt, "Nonalcoholic steatohepatitis: definition and pathology," Seminars in Liver Disease, vol. 21, no. 1, pp. 3-16, 2001.

[24] D. E. Kleiner, E. M. Brunt, M. Van Natta et al., "Design and validation of a histological scoring system for nonalcoholic fatty liver disease," Hepatology, vol. 41, no. 6, pp. 1313-1321, 2005.

[25] S. Chitturi, S. Abeygunasekera, G. C. Farrell et al., "NASH and insulin resistance: insulin hypersecretion and specific association with the insulin resistance syndrome," Hepatology, vol. 35, no. 2, pp. 373-379, 2002.

[26] G. Pagano, G. Pacini, G. Musso et al., "Nonalcoholic steatohepatitis, insulin resistance, and metabolic syndrome: further evidence for an etiologic association," Hepatology, vol. 35, no. 2, pp. 367-372, 2002.

[27] S. E. Shoelson, J. Lee, and A. B. Goldfine, "Inflammation and insulin resistance," Journal of Clinical Investigation, vol. 116, no. 7, pp. 1793-1801, 2006.

[28] C. P. Day and O. F. W. James, "Steatohepatitis: a tale of two "Hits"?" Gastroenterology, vol. 114, no. 4, pp. 842-845, 1998.

[29] J. D. Browning and J. D. Horton, "Molecular mediators of hepatic steatosis and liver injury," Journal of Clinical Investigation, vol. 114, no. 2, pp. 147-152, 2004.

[30] Y. Kamada, T. Takehara, and N. Hayashi, "Adipocytokines and liver disease," Journal of Gastroenterology, vol. 43, no. 11, pp. 811-822, 2008.

[31] N. M. W. De Alwis and C. P. Day, "Genetics of alcoholic liver disease and nonalcoholic fatty liver disease," Seminars in Liver Disease, vol. 27, no. 1, pp. 44-54, 2007.

[32] A. Rubio, E. Guruceaga, M. Vázquez-Chantada et al., "Identification of a gene-pathway associated with non-alcoholic steatohepatitis," Journal of Hepatology, vol. 46, no. 4, pp. 708718,2007

[33] W. Sato, Y. Horie, E. Kataoka et al., "Hepatic gene expression in hepatocyte-specific Pten deficient mice showing steatohepatitis without ethanol challenge," Hepatology Research, vol. 34, no. 4, pp. 256-265, 2006.

[34] Z. M. Younossi, A. Baranova, K. Ziegler et al., "A genomic and proteomic study of the spectrum of nonalcoholic fatty liver disease," Hepatology, vol. 42, no. 3, pp. 665-674, 2005.

[35] Y. Li, M. Komai-Koma, D. S. Gilchrist et al., "Galectin-3 is a negative regulator of lipopolysaccharide-mediated inflammation," Journal of Immunology, vol. 181, no. 4, pp. 27812789, 2008.
[36] D. K. Hsu, R. Y. Yang, Z. Pan et al., "Targeted disruption of the Galectin-3 gene results in attenuated peritoneal inflammatory responses," American Journal of Pathology, vol. 156, no. 3, pp. 1073-1083, 2000.

[37] E. Ip, G. Farrell, P. Hall, G. Robertson, and I. Leclercq, "Administration of the potent PPAR $\alpha$ agonist, Wy-14,643, reverses nutritional fibrosis and steatohepatitis in mice," Hepatology, vol. 39, no. 5, pp. 1286-1296, 2004.

[38] A. E. Feldstein, A. Canbay, P. Angulo et al., "Hepatocyte apoptosis and Fas expression are prominent features of human nonalcoholic steatohepatitis," Gastroenterology, vol. 125, no. 2, pp. 437-443, 2003.

[39] P. S. Ribeiro, H. Cortez-Pinto, S. Solá et al., "Hepatocyte apoptosis, expression of death receptors, and activation of NF$\kappa \mathrm{B}$ in the liver of nonalcoholic and alcoholic steatohepatitis patients," American Journal of Gastroenterology, vol. 99, no. 9, pp. 1708-1717, 2004.

[40] J. M. Schattenberg, P. R. Galle, and M. Schuchmann, “Apoptosis in liver disease," Liver International, vol. 26, no. 8, pp. 904$911,2006$.

[41] J. Ogawa, H. Kanegane, K. Tsuneyama et al., "Platelet-derived growth factor may be associated with fibrosis in a Down syndrome patient with transient myeloproliferative disorder," European Journal of Haematology, vol. 81, no. 1, pp. 58-64, 2008.

[42] J. S. Campbell, S. D. Hughes, D. G. Gilbertson et al., "Plateletderived growth factor $\mathrm{C}$ induces liver fibrosis, steatosis, and hepatocellular carcinoma," Proceedings of the National Academy of Sciences of the United States of America, vol. 102, no. 9, pp. 3389-3394, 2005.

[43] D. Cai, M. Yuan, D. F. Frantz et al., "Local and systemic insulin resistance resulting from hepatic activation of IKK- $\beta$ and NF«B," Nature Medicine, vol. 11, no. 2, pp. 183-190, 2005.

[44] A. Wieckowska, B. G. Papouchado, Z. Li, R. Lopez, N. N. Zein, and A. E. Feldstein, "Increased hepatic and circulating interleukin-6 levels in human nonalcoholic steatohepatitis," American Journal of Gastroenterology, vol. 103, no. 6, pp. 13721379, 2008.

[45] E. Bugianesi, N. Leone, E. Vanni et al., "Expanding the natural history of nonalcoholic steatohepatitis: from cryptogenic cirrhosis to hepatocellular carcinoma," Gastroenterology, vol. 123, no. 1, pp. 134-140, 2002.

[46] H. B. El-Serag and K. L. Rudolph, "Hepatocellular carcinoma: epidemiology and molecular carcinogenesis," Gastroenterology, vol. 132, no. 7, pp. 2557-2576, 2007.

[47] R. M. London and J. George, "Pathogenesis of NASH: animal Models," Clinics in Liver Disease, vol. 11, no. 1, pp. 55-74, 2007.

[48] Y. Nakanishi, K. Tsuneyama, M. Fujimoto et al., "Monosodium glutamate (MSG): a villain and promoter of liver inflammation and dysplasia," Journal of Autoimmunity, vol. 30, no. 1-2, pp. 42-50, 2008. 


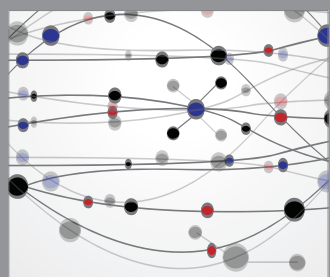

The Scientific World Journal
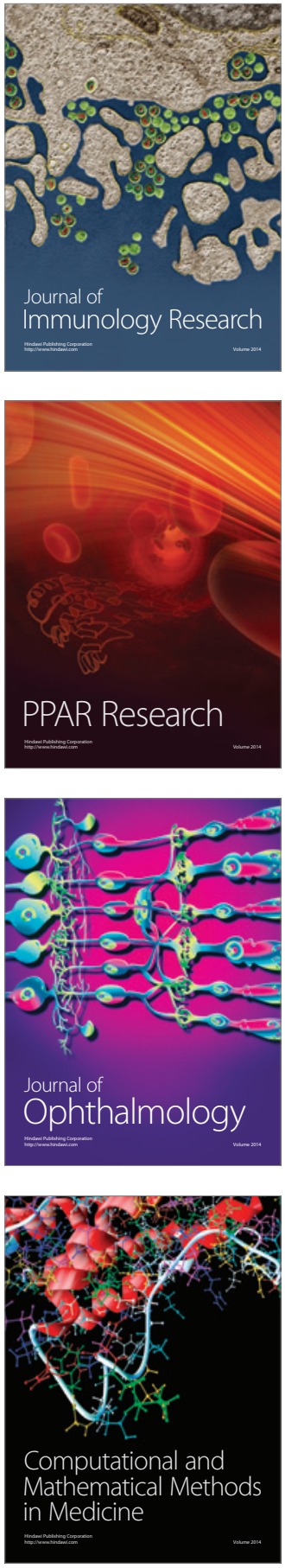

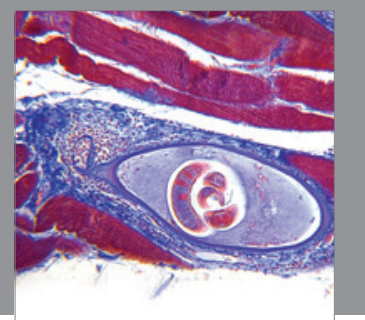

Gastroenterology

Research and Practice
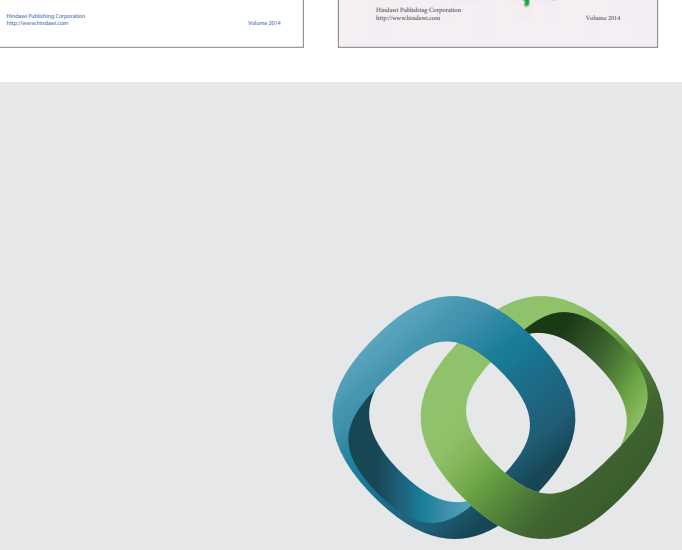

\section{Hindawi}

Submit your manuscripts at

http://www.hindawi.com
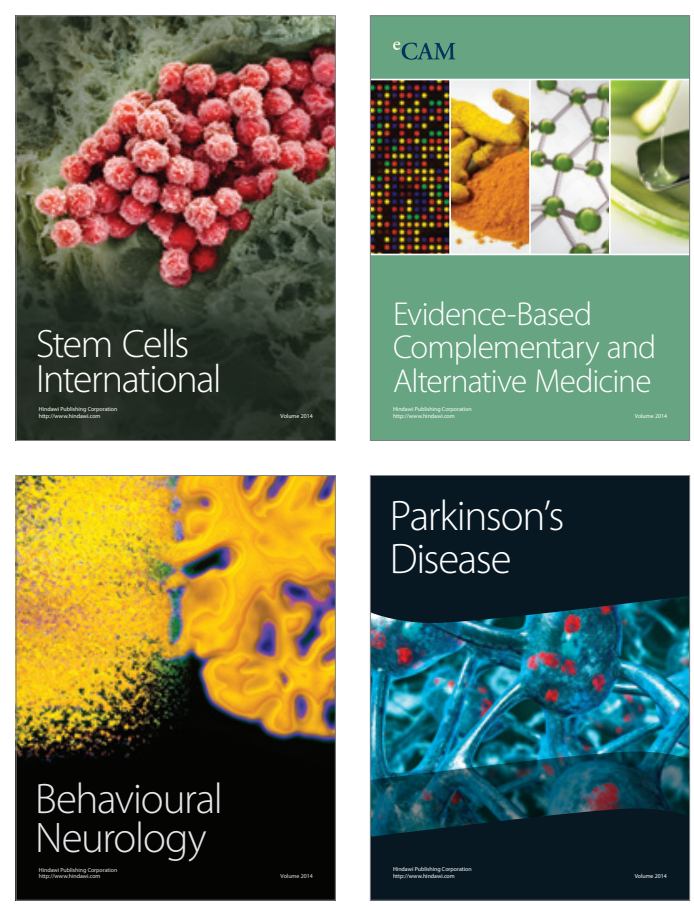

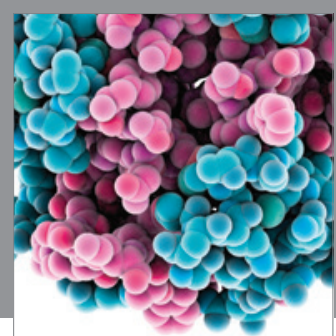

Journal of
Diabetes Research

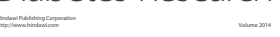

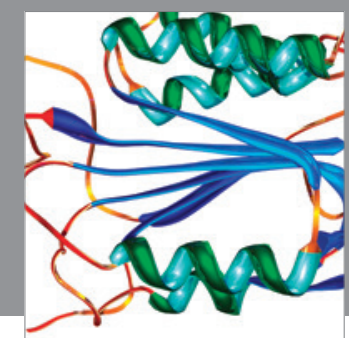

Disease Markers
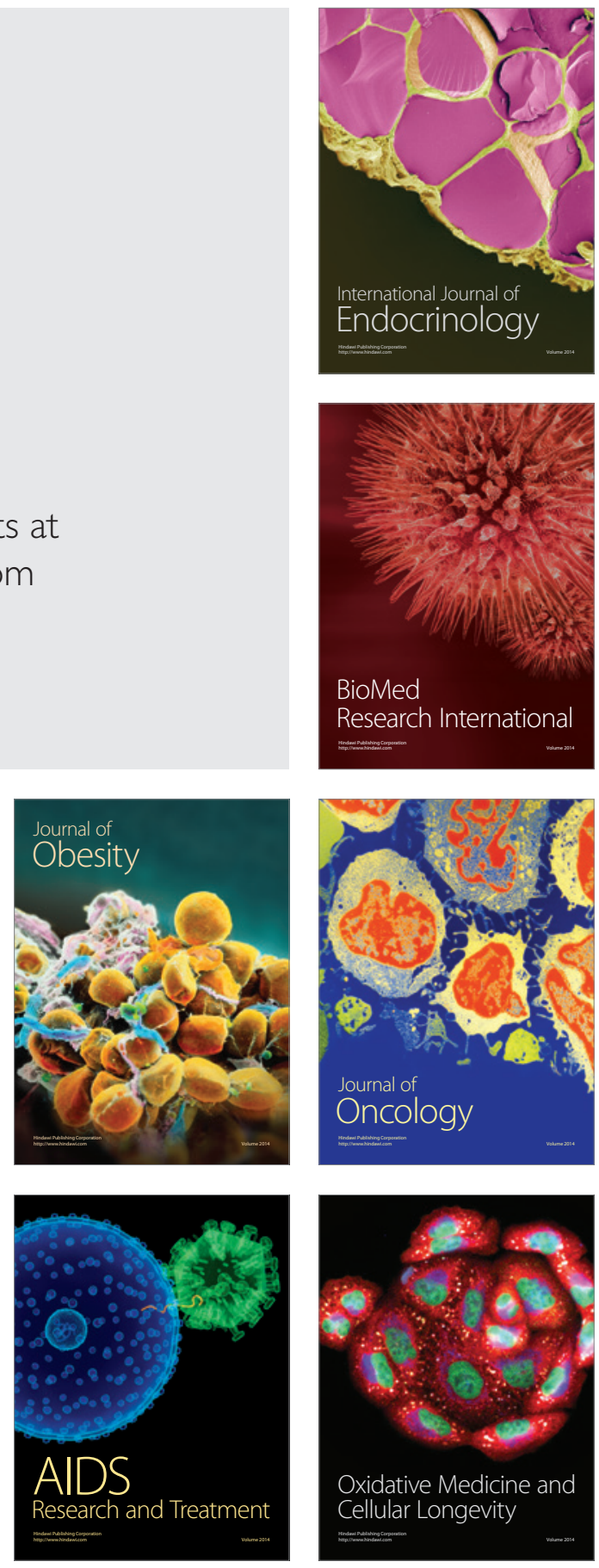